\title{
Disrupted Sleep During a Pandemic
}

Niraj Kumar, MD, DMa, Ravi Gupta, MD, PhD ${ }^{\text {, * * }}$

\section{KEYWORDS}

- Sleep • SARS-CoV-2 infection • COVID-19 • Insomnia • Poor quality sleep

\section{KEY POINTS}

- Sleep disturbances may be seen in up to three-fourth of COVID-19 patients, and up to two-fifth of health care workers and general population.

- Older age, having a partner alongside, and staying in a high-income country reduced the risk of sleep disturbances.

- Younger age, female sex, financial problems and coexisting stress, anxiety, and depression enhanced the risk of sleep disturbances.

- Etiology of sleep disturbances in COVID-19 pandemic is multifactorial. Three major factors neuroinvasion by SARS-CoV-2, social factors and health-policy changes during the pandemic can be implicated as contributors.

- A close association of sleep with immunity and metabolism means identification and treatment of sleep disturbances are essential during the pandemic time.

- Support from "Telemedicine sleep clinic" along with motivating the population to manage the zeitgebers may improve sleep during the pandemic.

\section{INTRODUCTION}

A pandemic is described as a state where a disease evolves quickly, affects a significant proportion of the population, and usually spreads across geographic boundaries to involve more than one continent. ${ }^{1,2}$ In the 21 st century, the world has suffered 3 pandemics: severe acute respiratory syndrome (SARS-CoV) in 2003, Swine Flu (H1N1) in 2009, and since late 2019, COVID-19 (SARS-CoV2). ${ }^{1-3}$ Among them, the COVID-19 pandemic had the most marked effect on health through direct and indirect factors, by inducing physiologic alteration across systems and by paving way for economic as well as political changes in a time of scientific uncertainty. ${ }^{2}$

Sleep is one of the important physiologic activities performed by all animal species. Contrary to prevalent belief of many, sleep is no longer considered as a period of inactivity by the sleep scientific community. Indeed, it is associated with several important functions required for a healthy life-rejuvenation, memory consolidation, modulation of immune function, and regulation of hormonal secretion. ${ }^{4,5}$ The pandemic has affected sleep in different ways. For a considerable proportion of the population, sleep has worsened in terms of quality, duration, and timing, but for a small proportion, it has improved. ${ }^{6}$ Sleep was affected by several factors during the COVID-19 pandemic. Unprecedented lockdowns limited zeitgebers (eg, physical activity, exposure to daylight, social rhythm, screen exposure, time of food intake) that regulate the sleep-wake cycle. Studies have also shown that a sizable number of people experience distress associated with uncertainties regarding

Credit Lines: All the illustrations (figures and tables) are drawn/prepared by the authors; hence, permission from a third party is not required.

a Department of Neurology, Division of Sleep Medicine, All India Institute of Medical Sciences, Rishikesh 249203, India; b Department of Psychiatry, Division of Sleep Medicine, All India Institute of Medical Sciences, Rishikesh 249203, India

* Corresponding author. Department of Psychiatry and Division of Sleep Medicine, All India Institute of Medical Sciences, Rishikesh 249203, India.

E-mail address: sleepdoc.ravi@gmail.com 
the course of treatment and outcome of SARSCoV-2 infection, as well as their employment. ${ }^{7}$ Together, these issues led to changes in sleep duration, timing, and quality in vulnerable individuals. ${ }^{7}$ For others, the lockdown was a respite that increased the sleep duration and improved sleep quality as suggested by reduction of social jet lag. . $^{8,9}$

Though the impact of pandemics on sleep has also been studied, it has been most extensively researched during the current COVID-19 pandemic. This article will present the available literature and will be divided into the following sections for better understanding.

1. Magnitude of sleep disturbances during the pandemic

2. Determinants and pathophysiology of sleep disturbances during COVID-19 pandemic

3. Long-term perspectives of sleep disturbances

4. Why is there a need to pay attention to sleep disorders during pandemics

5. Management of sleep disturbances during a pandemic

\section{MAGNITUDE OF SLEEP DISTURBANCES DURING THE PANDEMIC}

Sleep disturbances during pandemics have been reported across different age groups and in a variety of populations ranging from the general population and front-line workers to hospitalized and quarantined patients with SARS-CoV-2 infection. Available studies focused on the assessment of sleep quality, sleep-wake schedule, and insomnia.

The magnitude of sleep problems reported in studies is influenced by several factors: time at which the problem was assessed (lockdown vs after unlocking), age, gender, comorbidity, and medication history of subjects. It has been reported that detrimental changes in sleep pattern during early lockdown tend to remain the same unless early intervention is provided. ${ }^{10}$ Choice of questionnaires and measures used to assess sleep also affected the results across studies. For example, a higher prevalence of poor sleep quality has been reported from the studies using standardized measures of sleep like the Pittsburgh Sleep Quality Index (PSQI) compared with studies using other scales and investigator-developed measurements. ${ }^{11}$ Cut-off scores of the questionnaires also influenced the outcomes; the cut-off for the PSQI was higher than standard in some of the studies that could have resulted in lower prevalence. ${ }^{12} \mathrm{~A}$ summary of the available meta-analysis which addressed the prevalence of sleep disturbances during the COVID-19 pandemic is presented in Table 1.

\section{Sleep Quality}

\section{Among adults}

Sleep health includes 6 measures, namely regularity of sleep-wake schedule, duration of sleep, timing of sleep and wake, satisfaction with sleep, sleep efficiency, and daytime alertness. These are all indirect measures of sleep quality. ${ }^{17}$ Poor sleep health was reported by nearly one-fourth to half of the adult participants ranging between 18 and 94 years of age..$^{9,17}$ There was a geographic variation in sleep health, with poorer sleep health among residents of Latin American and Caribbean countries compared with residents of North America, Europe, and Central Asia. ${ }^{17}$ Owing to geographic variation, pooled analysis would provide better estimates of disturbed sleep among the general population. A systematic review reported that in the general population, nearly onethird had reported disturbed sleep. ${ }^{11}$

In a pooled analysis of sleep using questionnaires, sleep disturbance was shown to be higher among health care workers (HCWs) at nearly $40 \% .{ }^{11,16}$ Similar to the general population, geographic variation of sleep disturbance was also noted among HCWs; it was lower in China compared with other countries like Bahrain and Iraq. ${ }^{16}$ Contrary to intuitive deduction, it is difficult to reliably comment whether prevalence has actually increased among HCW during the pandemic as it appeared comparable to pre-pandemic time at least by one at study group. $^{11}$

A systematic review of cross-sectional studies reported that sleep quality was poor during the pandemic, not only in HCWs (irrespective of their engagement in the management of COVID-19 patients during the pandemic) but also among nonhospitalized individuals (irrespective of the status of SARS-CoV-2 infection). ${ }^{18}$ Interestingly, in most of the studies, poor sleep quality was reported despite spending longer time in bed. ${ }^{18}$ This is a situation similar to paradoxic insomnia wherein despite an adequate sleep duration, sleep is still nonrefreshing, perhaps owing to increased cortical activation. ${ }^{19}$

Patients with SARS-CoV-2 infection make the largest proportion of the group in which sleep quality was poor during pandemic (nearly three-fourth reported). ${ }^{11}$ This is in contrast to another metaanalysis, which reported that only $34 \%$ of patients having SARS-CoV-2 infection had disturbed sleep or poor quality sleep. ${ }^{12}$ In the latter paper, most of the studies were from China, whereas the earlier paper represented better global prevalence because it included studies from various countries.

Contrary to reports of poor or worsening sleep quality in most studies, a minority of the population 


Table 1
Prevalence of sleep disturbances during CovID-19 pandemic across meta-analyses

(6\%) reported improvement of sleep during the pandemic. ${ }^{6}$ With one study reporting nearly a quarter of subjects having improvement in sleep quality during the lockdown irrespective of the total sleep time at night. ${ }^{9}$

Taken altogether, the available data show that geographic variation exists among subjects reporting poor sleep quality both in the general population and in patients suffering from SARSCoV-2 infection. These factors could also emphasize the role of genetics in their vulnerability (eg, MEIS1 gene has been found to be associated with insomnia, MEIS1 and BTBD-9 have been found to increase the risk for restless legs syndrome, etc.) to develop sleep disturbances in association with environmental factors. ${ }^{7}$

\section{Among children and adolescents}

Poor and worsening quality of sleep was observed among nonhospitalized children and adolescents from Spain and India. ${ }^{20,21}$ Dutta and colleagues reported worsening of sleep among a third of children in India. ${ }^{21}$ However, sleep quality did not take a downhill course throughout the pandemic. It seemed to worsen in the initial phase of lockdown and thereafter remained stable. ${ }^{10}$ And compared with adults, a greater proportion of children (43\%) reported deeper sleep during the lockdown. ${ }^{21}$

\section{Sleep-Wake Schedule}

\section{Among adults}

Two major shifts in the sleep-wake schedule were reported during the COVID-19 pandemicdelayed sleep phase and extension of sleep time. A shift to a later bedtime and later wake time was reported by nearly $50 \%$ to $60 \%$ of the adult population in an online survey conducted across 59 countries. ${ }^{17}$ However, figures vary across studies and a study from India reported that only a third of the subjects had delayed sleep-wake schedules. ${ }^{9}$ In Canada, $63 \%$ of subjects had earlier bedtime and later wake time during the lockdown, whereas only $2 \%$ of the population 
reported the same from India., ${ }^{6,9}$ This suggested an extension of bedtime that had occurred during the pandemic in some of the population. Extension of sleep resulted in reduction of social jet lag during lockdowns and maintained consistent circadian rhythm across weeks. ${ }^{8}$

Difficulty in sleep initiation (approximately 39\%) and in maintaining sleep (32\%) was reported by the adult population during the COVID-19 pandemic. ${ }^{17}$ Although difficulty in sleep initiation resulted in delayed awakening, prominent problems in sleep maintenance and early morning awakenings resulted in reduced total sleep time. ${ }^{6}$

\section{Among children and adolescents}

There is no meta-analysis available on sleep-wake schedule for this population. Findings across different individual studies, on the other hand, appeared to contradict one another. One study reported a change to a later bedtime and wake time on weekdays, but not on weekends (which were already delayed even before lockdown). ${ }^{21}$ The delayed bedtime and delayed wake time were reported among children aged 3 to 16 years. ${ }^{22,23}$ This could be related to the prolongation of sleep-onset latency, both on weekdays and weekends. ${ }^{21,23}$ Similarly, the frequency of napping also increased after lockdown in one study, whereas reduced in another. ${ }^{21,23}$ Interestingly, the sleepwake changes did not develop to a free-running type of schedule. Rather, it worsened for an initial period after lockdown and remained stable thereafter. ${ }^{10}$

\section{Duration of Sleep}

\section{Among adults}

On average, a total sleep duration of 7 hours was reported among adults during the pandemic. ${ }^{6,17}$ Those who used to sleep shorter than 6 to 8 hours before pandemic had reduced sleep duration pandemic, whereas those who usually slept for 6 to 8 hours during pre-pandemic period, did not report any change in either their sleep duration or had an increase in time spent asleep. ${ }^{17}$

\section{Among children and adolescents}

In children aged 3 to 18 years, there was an increase in the total sleep time. ${ }^{13,23}$ In one study, half of the children were sleeping 12 hours a day, and only one-fourth were sleeping less than 8 hours a day. ${ }^{22}$ Being cross-sectional in design, this study was not able to comment on the change in sleep duration. There was an increase in total sleep time on weekdays, but there was no change on weekends reported after lockdown. ${ }^{21}$ This also varies geographically, with a greater change in Italy compared with Spain. ${ }^{24}$

\section{Groupings Based on Change in Sleep-Wake Schedule}

Based on the change in sleep-wake schedule during pandemic, 3 populations were identified in one study-extended time in bed (63\% subjects), reduced time in bed (13\%), and delayed sleep (24\%). ${ }^{6}$ Fewer work responsibilities and working from home resulted in delayed sleep. ${ }^{6}$ Perhaps these people were able to follow their own sleepwake schedules because of lack of compulsion to adhere to a structured routine. Gupta and colleagues ${ }^{9}$ identified 3 groups in a population based survey-reduced sleep duration (16\%), extended sleep duration (18\%), and participants where total sleep time remained unchanged $(66 \%)$. Similar to results of above mentioned study, nearly equal proportion of participants (28\%) in this study fell into the "delayed sleep" category. Subjects in the reduced sleep group reported prolonged sleep onset latency and depressive features. ${ }^{9}$ Thus, the available data suggest that sleep schedule vary across studies. The lower prevalence in the Gupta and colleagues study could be related to the inclusion of HCWs in the study population. ${ }^{9}$ These findings also emphasize the role of social factors and structured routine as a zeitgeber. ${ }^{25}$

\section{Sleep Disorders During the Lockdown}

Among healthy adults, the prevalence of insomnia during lockdown appeared to remain similar to the prelockdown period. ${ }^{9}$ Yet sleep physicians report an increase in the number of patients seeking help for insomnia and delayed sleep-wake phase sleep disorders. ${ }^{26}$ Underlying factors are not known but could be related to the availability of teleconsultation facilities, ability to spare time for health issues, and increased awareness of health issues in view of the prevailing situation.

Contrary to adults, sleep disorders increased among children. There was an increase in difficulty falling asleep, night-time awakenings, nightmares, sleep terrors, and excessive daytime sleepiness in the preadolescent age group. ${ }^{23}$

\section{DETERMINANTS AND PATHOPHYSIOLOGY OF SLEEP DISTURBANCES}

Several factors were responsible for clinically significant sleep disturbances during the pandemic (Table 2). Interestingly, the presence of anxiety (threat perception) among parents lessened the sleep time of children, suggesting the role of shared genes as well as the environment in the occurrence of anxiety. ${ }^{24}$ 
Table 2

Protective and detrimental factors related to sleep

\section{Protective}

Older age ${ }^{17}$

Having a partner ${ }^{17}$

Living in high-income

countries $^{17}$

Detrimental
Younger age $^{11}$
Good sleep before pandemic $^{6}$
Women $^{6}$
Being employed $^{6}$
Financial problems $^{17}$
Family responsibilities $^{6}$
Chronic diseases $^{6}$
Stress and anxiety $^{6,17,24}$
Depression
Increased alcohol $^{6}$ (consumption
$\quad$ ${ }^{6,17}$
Stricter lockdown
Longer screen time $^{17}$
Physical inactivity in previously
active persons

\section{Directly Related to the IIIness}

COVID-19 can cause sleep disruption due to nervous system invasion or systemic (non-neural) invasion along with cytokine storm (Fig. 1).

\section{Nervous system invasion}

Several potential pathways of neuro-invasion have been suggested for SARS-CoV-2. It may have a trans-synaptic spread where the virus retrogradely travels to the CNS after entering the peripheral nerve terminals of its primary target organs viz., oronasal mucosa and lungs. From nasal mucosa, it may spread through cribriform plate and olfactory pathways to reach the cerebral cortex. SARS-CoV-2 can also enter CNS by crossing the blood-brain barrier (BBB) through one of the two routes-either by infecting and traveling across vascular endothelium or by infecting leukocytes, which then cross the BBB ("Trojan Horse"

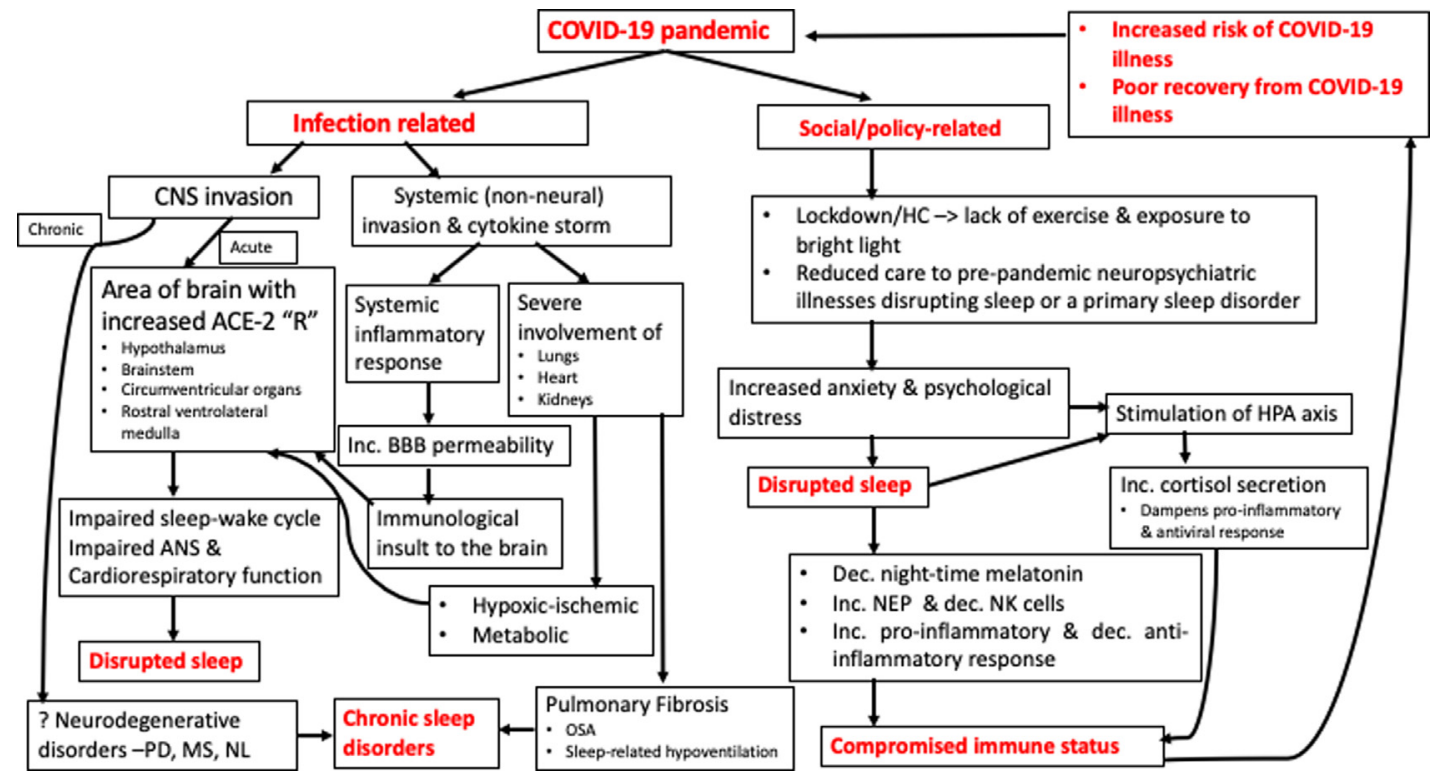

Fig. 1. Pathophysiology of sleep disruption during the COVID-19 pandemic. ACE-2 "R", angiotensin-converting enzyme-2 receptor; ANS, autonomic nervous system; BBB, blood-brain barrier; CNS, central nervous system; COVID-19, coronavirus disease 2019; Dec., decreased; HC, home confinement; HPA, hypothalamic-pituitaryadrenal axis; Inc., increased; MS, multiple sclerosis; NEP, norepinephrine; NK cells, natural killer cells; NL, narcolepsy; OSA, obstructive sleep apnea; PD, Parkinson disease. 
mechanism). ${ }^{28}$ Once inside the brain, SARS-CoV2 invades tissues with ACE- 2 receptors including the frontotemporal cortex, circumventricular organs, thalamus, and brainstem involving the rostral ventrolateral medulla among others. ${ }^{28,29}$ ACE-2 expression has also been reported in the hypothalamus and pituitary gland. ${ }^{30}$ Involvement of the hypothalamus and the brainstem may result in impairment of sleep-wake cycle regulation and dysautonomia with cardiorespiratory dysfunction, thereby, resulting in poor sleep quality presenting as insomnia and sleep-disordered breathing. ${ }^{31}$

\section{Systemic invasion}

Although the primary involved organ in SARSCoV-2 is the lungs, other organs including the heart and kidneys may also be infected causing cardiorespiratory and metabolic derangements. The former may result in hypoxic-ischemic events and the latter causes metabolic encephalopathy. ${ }^{28}$ In addition, a systemic inflammatory response may increase the BBB permeability, thereby, predisposing the brain to immunologic insult from a cytokine storm, which may precipitate neuropsychiatric features. ${ }^{28,32}$ This, in turn, may result in sleep disruption.

In addition, the possibility of pulmonary fibrosis after severe COVID pneumonia may result in OSA and sleep-related hypoventilation as a longterm post-COVID complication. ${ }^{33}$ Systemic hyperinflammation leading to deranged immunity in COVID-19 may lead to BBB compromise and involvement of the gut-brain axis, thereby, raising the possibility of developing chronic neuroinflammatory and neurodegenerative disorders such as Parkinson disease, multiple sclerosis, narcolepsy, and associated sleep disorders. ${ }^{34}$

\section{Indirectly Related to COVID-19}

\section{Social factors}

During the pandemic, COVID-19 patients are confined in hospitals or quarantined in their homes/facilities. Most of the noninfected population remain restricted in their homes. COVID-19 has been linked to increased anxiety and psychological distress in affected patients. Psychological distress may result from physical discomfort, hospitalization in an unknown scenario among unacquainted caregivers, and uncertainty in the course of illness. ${ }^{32,35,36}$ In fact, disturbed mental health and long-term neuropsychiatric sequelae may follow COVID-19 illness in vulnerable patients. $^{37}$ Stress and mental ill-health, in turn, disrupt sleep. ${ }^{38}$ Noninfected population also reports significant anxiety, stress, and sleep disruption. ${ }^{9,35,39,40}$ This may be due to disrupted circadian rhythm owing to lack of exposure to bright light, change in sleep-wake pattern, reduced physical exercise, unpredictability regarding health and economic condition in the general population confined at home. ${ }^{41}$

\section{Relation to changes in health policies}

From the onset of this pandemic, focus of the health system shifted toward COVID-19, and rightly so. But this significantly impacted the availability of medical care for patients suffering from other medical disorders including those with prepandemic neuropsychiatric illnesses. ${ }^{31}$ In fact, patients with prepandemic neuropsychiatric illnesses such as Parkinson disease reported significant sleep disturbances. ${ }^{42}$ The pandemic significantly affected the "Sleep Medicine" practice and influenced care of patients having sleep disorders even before the pandemic. ${ }^{43} \mathrm{~A}$ significant reduction in laboratory-based continuous positive airway pressure or bilevel positive airway pressure (PAP) titration studies was reported. ${ }^{43}$ Owing to the risk of aerosol generation, the use of PAP was even discouraged. ${ }^{44}$ These changes in health policies could have resulted in increased anxiety and psychological distress and contributed to further worsening of sleep in patients with preexisting pre sleep disorders.

Increased anxiety and psychological distress activate the hypothalamus-pituitary-adrenal axis and stimulate cortisol secretion. Sleep disruption resulting from increased anxiety and psychological distress may further worsen stress, forming a vicious cycle between stress and sleep disruption. ${ }^{45}$ The resulting hypercortisolism dampens the proinflammatory pathways and antiviral immunity suffers. Increased norepinephrine levels in sleep-disrupted patients suppress natural killer cells, thereby, further compromising the immune status. ${ }^{46-48}$ Reduced night-time melatonin, observed in patients with disrupted sleep, may deprive the patient of its anti-inflammatory, antioxidant, and immunomodulatory properties, thereby increasing risk of infection there. ${ }^{49,50}$

Thus, the etiology of sleep disturbances during COVID-19 appears multifactorial. If left unattended, sleep deprivation may result in enhanced functioning of proinflammatory cytokines including interleukin (IL)-1, IL-6, and tumor necrosis factoralpha; and suppression of anti-inflammatory cytokines including IL-10. ${ }^{51,52}$ This may predispose patients to inflammatory disorders by enhancing the expression of proinflammatory genes. ${ }^{52}$ By affecting antioxidant enzymes, long-term sleep deprivation may also precipitate oxidative stress. ${ }^{53}$ Unmanaged sleep abnormalities in the general population increase the risk of COVID-19 and can negatively influence the recovery of those 
infected. Thus, sleep disruption during the pandemic appears to play an essential role in the propagation of COVID-19.

\section{Effect of pandemic on patients with existing sleep disorders}

There is an increase in the risk of contracting COVID-19 infection among patients with sleep disorders, especially OSA. The pandemic led to the closure of sleep medicine facilities and a sharp decline in available services, leaving patients with sleep disorders without treatment. ${ }^{26,43,54,55}$ In time, many centers chose to serve their patients via telemedicine and provided limited treatment. ${ }^{26,43,55}$ The pandemic has become a boon for the development of telemedicine facilities and many physicians prefer to continue even after the pandemic is over. ${ }^{26}$ This could reduce the cost of care for patients, especially indirect costs incurred toward the treatment.

\section{LONG-TERM PERSPECTIVES OF SLEEP DISORDERS IN VIEW OF PANDEMIC}

As discussed in the pathophysiology section, SARS-CoV-2 infection may leave a permanent scar in the form of neuropsychiatric sequela. Rogers and colleagues ${ }^{37}$ reported that $12 \%$ of subjects reported insomnia as long as 24 months after recovery from SARS and MERS infection. Insomnia has been reported in nearly one-third of the patients even after months of recovery from SARS-CoV-2 infection. ${ }^{56}$

Literature derived from SARS infection shows that nearly a quarter of patients suffer from anxiety, depressive feelings, and post-traumatic stress disorder (PTSD) during acute infection until after 6 months. ${ }^{57}$ These disorders are frequently associated with disturbed sleep, especially insomnia. Other sleep disorders, for example, restless legs syndrome, parasomnia may arise in association with psychotropics used for the treatment of these disorders. HCWs were likewise found to have anxiety, depressive features, and PTSD during and after the SARS pandemic. ${ }^{57}$ Hence, there is a need to screen HCWs for sleep disturbances and sleep disorders even after the COVID-19 pandemic is over.

\section{WHY IS THERE A NEED TO PAY ATTENTION TO SLEEP DISORDERS DURING PANDEMICS?}

Several factors emphasize the need to pay attention to sleep. First, systemic inflammatory changes and sleep disturbances run in a vicious cycle. To interrupt it, attention should be paid to reduce systemic inflammation as well as restore good quality and optimal sleep. Second, vaccination is considered a major weapon against the SARSCOV-2 infection. Sleep deprivation reduces the immune response to vaccines ${ }^{58}$ and optimal duration of sleep is important for mounting an adequate immunologic response after vaccination. Howev$\mathrm{er}$, data regarding sleep quality are not available. ${ }^{58}$ Third, timing of vaccination is important as immune response follows a circadian pattern, being greater if vaccine is given in the morning hours. ${ }^{58}$ Fourth, subjects with OSA are at higher risk for complications from SARS-COV-2 infection such as admission to intensive care units and death. ${ }^{54,59}$ It is a known fact that obesity is a risk factor for OSA. Increased production of cytokines, reduced adiponectin, endothelial dysfunction, activation of prothrombotic cascade, ectopic lipid in alveolar type 2 cells, and insulin resistance have been proposed to worsen course of SARS-COV-2 infection among these individuals. ${ }^{60}$ OSA has been found to worsen many of these parameters, adding the risk reported in earlier studies.

\section{MANAGEMENT OF SLEEP DISTURBANCES AND SLEEP DISORDERS}

In response to the COVID-19 pandemic, several medical societies have proposed guidelines to improve sleep for patients, strategies to manage sleep disorders, and continue sleep-related services for patients during the lockdown. Table 3 summarizes these published guidelines.

Sleep disturbances during the lockdown were multifactorial and occurred only in predisposed subjects, as already discussed. As sleep-wake schedules have changed in a large proportion of the population, it has been advised that maintenance of proper routine and sleep hygiene practices needs to be followed even during lockdown. ${ }^{68}$ Timing of intervention is important. Early intervention is more useful for prevention and can be achieved through dissemination of educational information, whereas intervention initiated in the later period needs to be more structured and individualized. ${ }^{10}$

Social jet lag was shown to be reduced during the lockdown among subjects who discontinued work or were working from home. ${ }^{8}$ There is a need to educate the population regarding the importance of adequate sleep duration and manage work schedules irrespective of prevailing situations. This will help lessen sleep deprivation and boost immunity. Time saved from commuting and reduced workload may be used to increase daylight exposure, exercise, and social communication, and cultivate hobbies. All these factors are known to improve sleep quality. ${ }^{39,69}$ 


\section{Table 3}

Published literature for sleep medicine practice during the pandemic

\begin{tabular}{lll} 
S.N. & Title & Society \\
\hline 1. & $\begin{array}{c}\text { Considerations for the practice } \\
\text { of sleep medicine during } \\
\text { COVID-1961 }\end{array}$ & $\begin{array}{c}\text { American Academy of Sleep } \\
\text { Medicine }\end{array}$ \\
& &
\end{tabular}

Issues Addressed

- COVID-19 testing before sleep study

- Home Sleep Apnea Test (HSAT) and In-lab testing

- PAP therapy

- Mitigating risk of personnel, facility, and equipment
2. Dealing with sleep problems during home confinement due to the COVID-19 outbreak: Practical recommendations from a task force of the European CBT-I Academy ${ }^{39}$

\section{European CBT-I Academy}

Nonpharmacological methods for:

- Improving sleep during home confinement

- Recommendations for women and children

- Recommendations for health care workers and those with increased burden

- Medications to improve sleep

\section{Guidelines of the Indian Society for Sleep Research (ISSR) for Practice of Sleep Medicine during COVID- $19^{62}$}

\author{
Indian Society for Sleep \\ Research (ISSR)
}

- Telemedicine consultation:

- who can be provided teleconsultation, methods, advantages and limitations, legal issues, online prescriptions

- Sleep study during COVID19: safety of staff and patients, indications for HSAT and in-lab testing

- PAP therapy during COVID19: Current PAP users, titration methods at home and in-laboratory, temporary auto-PAP therapy in high-risk patients

4. Sleep laboratories reopening and COVID-19: a European perspective $^{63}$
European Respiratory Society and National Societies

- Conducting diagnostic study for OSA

- Protocol for titration study

- Protocol for patients already using PAP

- Recommendation for pediatric sleep studies

5. The Society of Behavioral Sleep Medicine (SBSM) COVID-19 Task Force: Objectives and Summary Recommendations for Managing Sleep during a Pandemic $^{64}$

6. Helping Canadian health care providers to optimize Sleep Disordered Breathing management for their patients during the COVID19 pandemic $^{65}$
Society of Behavioral Sleep Medicine

Canadian Thoracic Society
- Managing acute insomnia

- Managing irregular/delayed sleep-wake schedule

- Managing nightmares

- Considerations for children and elders

- Outpatients visit and sleep study

- Patients using PAP at home

- Patients with OSA using PAP while admitted to hospital

- Newly diagnosed OSA patients where PAP is required 
Table 3

(continued)

S.N. Title

Society

Issues Addressed

7. Sleep Breathing Disorders in

Italian Thoracic Society

- Diagnostic Sleep Study

the COVID-19 Era: Italian

- Initiation of PAP therapy

Thoracic Society

Organizational Models for a

- Follow-up of patients with

Correct Approach to OSA

Diagnosis and Treatment ${ }^{66}$

8. Restoring Pulmonary and

Sleep Services as the COVID-

19 Pandemic Lessens. From

an Association of

Pulmonary, Critical Care, and Sleep Division Directors

and American Thoracic

Society-coordinated Task Force $^{67}$
Association of Pulmonary,

Critical Care, and Sleep

Division Directors

American Thoracic Society
- Guidelines for resuming outpatient services

- Polysomnography services

- Other respiratory services like bronchoscopy, pulmonary function testing
As OSA is a risk factor, at least for contracting SARS-CoV-2 infection with potentially poor outcomes, patients should be screened for OSA. The 5 items of the Sleep Symptoms Scale can be used and high-risk patients may be advised home sleep apnea testing (HSAT), attended polysomnography, mitigation strategies, or titration with PAP device as per prevailing guidelines. ${ }^{61,70}$ Exercise has also been found to improve the apnea-hypopnea index. ${ }^{69}$ Hence, this may be advised to high-risk persons.

Reduction of exposure to negative information, particularly related to COVID-19, scheduling time to introspect and identify stress, sharing the stress with members of the family to identify possible solutions, reduction and sharing of burden for daily chores, and engagement in relaxing activities before bedtime have been recommended to improve sleep. ${ }^{39}$

Though cognitive behavior therapy for insomnia is recommended as the first-line therapy, its availability is an issue. ${ }^{39,71}$ In such cases, hypnotic medications may be given according to available guidelines. ${ }^{71,72}$

\section{SUMMARY}

During the COVID-19 pandemic, sleep disturbance increased in selected groups. Owing to the closure of sleep medicine services, patients had limited access to appropriate care and telemedicine became a viable alternative. Several sleep societies developed guidelines to improve sleep health in different populations and to adapt the practice of sleep medicine during the pandemic. Sleep disturbances were also shown to have a multifactorial origin. Evidence from basic sciences and clinical literature suggests that sleep disturbances can run a chronic course, and there is a need to educate people about sleep disturbances during the pandemic. Lastly, patients suffering from SARS-CoV-2 infection should also be screened and treated for sleep disorders during the acute phase and after recovery.

\section{CLINICS CARE POINTS}

- All patients should be screened for sleep quality and sleep patterns during the pandemic time

- Knowledge about sleep hygiene rules should be offered to all patients

- Patients with existing sleep disorders or at high risk for sleep disorders should be referred to sleep physicians for the management of sleep disorders

\section{DISCLOSURE}

The authors have nothing to disclose.

\section{REFERENCES}

1. Swetha G, Eashwar VMA, Gopalakrishnan S. Epidemics and pandemics in India throughout history: a review article. Indian J Public Health Res Dev 2019;10(8).

2. Huremović D. Brief history of pandemics (pandemics throughout history). Psychiatry of pandemics: A Mental Health Response to Infection Outbreak, 2019. Cham, Switzerland: Springer Nature; 2019. p. 7-35.

3. Cherry JD, Krogstad P. SARS: The first pandemic of the 21st century. Pediatr Res 2004;56(1):1-5. 
4. Reinke H, Asher G. Crosstalk between metabolism and circadian clocks. Nat Rev Mol Cell Biol 2019; 20(4):227-41.

5. Pocivavsek A, Rowland LM. Basic neuroscience illuminates causal relationship between sleep and memory: translating to schizophrenia. Schizophr Bull 2018;44(1):7-14.

6. Robillard R, Dion K, Pennestri MH, et al. Profiles of sleep changes during the COVID-19 pandemic: demographic, behavioural and psychological factors. J Sleep Res 2021;30(1):e13231.

7. Morin CM, Carrier J, Bastien C, et al. Sleep and circadian rhythm in response to the COVID-19 pandemic. Can J Public Health 2020;111(5):654-7.

8. Leone MJ, Sigman M, Golombek DA. Effects of lockdown on human sleep and chronotype during the COVID-19 pandemic. Curr Biol 2020;30(16):R930-1.

9. Gupta R, Grover S, Basu A, et al. Changes in sleep pattern and sleep quality during COVID-19 lockdown. Indian J Psychiatry 2020;62(4):370-8.

10. Dellagiulia A, Lionetti F, Fasolo M, et al. Early impact of COVID-19 lockdown on children's sleep: a 4-week longitudinal study. J Clin Sleep Med 2020;16(9): 1639-40.

11. Jahrami $H$, BaHammam AS, Bragazzi $N L$, et al. Sleep problems during the COVID-19 pandemic by population: a systematic review and meta-analysis. J Clin Sleep Med 2021;17(2):299-313.

12. Deng J, Zhou F, Hou W, et al. The prevalence of depression, anxiety, and sleep disturbances in COVID-19 patients: a meta-analysis. Ann N Y Acad Sci 2021;1486(1):90-111.

13. Panda PK, Gupta J, Chowdhury SR, et al. Psychological and behavioral impact of lockdown and quarantine measures for COVID-19 pandemic on children, adolescents and caregivers: a systematic review and meta-analysis. J Trop Pediatr 2021; 67(1):fmaa122.

14. Pappa S, Ntella V, Giannakas T, et al. Prevalence of depression, anxiety, and insomnia among healthcare workers during the COVID-19 pandemic: a systematic review and meta-analysis. Brain Behav Immun 2020;88:901-7.

15. Salari N, Khazaie H, Hosseinian-Far A, et al. The prevalence of sleep disturbances among physicians and nurses facing the COVID-19 patients: a systematic review and meta-analysis. Global Health 2020; 16(1):92.

16. Marvaldi M, Mallet J, Dubertret C, et al. Anxiety, depression, trauma-related, and sleep disorders among healthcare workers during the COVID-19 pandemic: a systematic review and meta-analysis. Neurosci Biobehav Rev 2021;126:252-64.

17. Yuksel D, Mckee GB, Perrin PB, et al. Sleeping when the world locks down: correlates of sleep health during the COVID-19 pandemic across 59 countries. Sleep Health 2021;7(2):134-42.
18. Souza LFF, Paineiras-Domingos LL, MeloOliveira MES, et al. The impact of COVID-19 pandemic in the quality of sleep by Pittsburgh Sleep Quality Index: a systematic review. Cien Saude Colet 2021;26(4):1457-66.

19. Rezaie L, Fobian AD, McCall WV, et al. Paradoxical insomnia and subjective-objective sleep discrepancy: a review. Sleep Med Rev 2018;40:196-202.

20. Lavigne-Cerván R, Costa-López B, Juárez-Ruiz De Mier R, et al. Consequences of COVID-19 confinement on anxiety, sleep and executive functions of children and adolescents in Spain. Front Psychol 2021;12:565516.

21. Dutta K, Mukherjee R, Sen D, et al. Effect of COVID19 lockdown on sleep behavior and screen exposure time: an observational study among Indian school children. Biol Rhythm Res 2020;1-12.

22. Ranjbar K, Hosseinpour H, Shahriarirad R, et al. Students' attitude and sleep pattern during school closure following COVID-19 pandemic quarantine: a web-based survey in south of Iran. Environ Health Prev Med 2021;26(1):33.

23. Bruni O, Malorgio E, Doria M, et al. Changes in sleep patterns and disturbances in children and adolescents in Italy during the Covid-19 outbreak. Sleep Med 2021. S1389-9457(21)00094-0.

24. Orgilés M, Morales A, Delvecchio E, et al. Immediate psychological effects of the COVID-19 quarantine in youth from Italy and Spain. Front Psychol 2020;11: 579038.

25. Mistlberger R, Skene D. Social influences on mammalian circadian rhythms: animal and human studies. Biol Rev Camb Philos Soc 2004;79(3): 533-56.

26. Kanchan S, Saini LK, Daga R, et al. Status of the practice of sleep medicine in India during COVID-19 pandemic. J Clin Sleep Med 2021;17(6):1229-35.

27. Martínez-De-Quel Ó, Suárez-Iglesias D, LópezFlores M, et al. Physical activity, dietary habits and sleep quality before and during COVID-19 lockdown: a longitudinal study. Appetite 2021;158: 105019.

28. Desai I, Manchanda R, Kumar N, et al. Neurological manifestations of coronavirus disease 2019: exploring past to understand present. Neurol Sci 2021;42(3):773-85.

29. Tremblay ME, Madore C, Bordeleau M, et al. Neuropathobiology of COVID-19: the role for glia. Front Cell Neurosci 2020;14:592214.

30. Pal R, Banerjee M. COVID-19 and the endocrine system: exploring the unexplored. J Endocrinol Invest 2020;43(7):1027-31.

31. Gupta R, Pandi-Perumal SR. SARS-CoV-2 infection: paving way for sleep disorders in long term. Sleep Vigil 2021;17:1-2.

32. Guo Q, Zheng Y, Shi J, et al. Immediate psychological distress in quarantined patients with COVID-19 
and its association with peripheral inflammation: a mixed-method study. Brain Behav Immun 2020; 88(January): 17-27.

33. Miller MA, Cappuccio FP. A systematic review of COVID-19 and obstructive sleep apnoea. Sleep Med Rev 2021;55:101382.

34. Schirinzi T, Landi D, Liguori C. COVID-19: dealing with a potential risk factor for chronic neurological disorders. J Neurol 2021;268(4):1171-8.

35. Wu KK, Chan SK, Ma TM. Posttraumatic stress, anxiety, and depression in survivors of severe acute respiratory syndrome (SARS). J Trauma Stress 2005; 18(1):39-42.

36. Silverstone PH. Prevalence of psychiatric disorders in medical inpatients. J Nerv Ment Dis 1996;184(1): 43-51.

37. Rogers JP, Chesney E, Oliver D, et al. Psychiatric and neuropsychiatric presentations associated with severe coronavirus infections: a systematic review and meta-analysis with comparison to the COVID19 pandemic. Lancet Psychiatry 2020;7(7):611-27.

38. Van Reeth $\mathrm{O}$, Weibel L, Spiegel K, et al. Interactions between stress and sleep: from basic research to clinical situations. Sleep Med Rev 2000;4(2):201-19.

39. Altena E, Baglioni C, Espie CA, et al. Dealing with sleep problems during home confinement due to the COVID-19 outbreak: practical recommendations from a task force of the European CBT-I Academy. J Sleep Res 2020;29(4):e13052.

40. Voitsidis P, Gliatas I, Bairachtari V, et al. Insomnia during the COVID-19 pandemic in a Greek population. Psychiatry Res 2020;289:113076.

41. de Sousa Martins e Silva E, Ono BHVS, Souza JC. Sleep and immunity in times of COVID-19. Rev Assoc Med Bras (1992) 2020;66(Suppl 2):143-7.

42. Kumar N, Gupta R, Kumar H, et al. Impact of home confinement during COVID-19 pandemic on Parkinson's disease. Parkinsonism Relat Disord 2020;80: 32-4.

43. Grote L, Mcnicholas WT, Hedner J. Sleep apnoea management in Europe during the COVID-19 pandemic: data from the European sleep apnoea database (ESADA). Eur Respir J 2020;55(6): 2001323.

44. Barker J, Oyefeso O, Koeckerling D, et al. COVID19: community CPAP and NIV should be stopped unless medically necessary to support life. Thorax 2020;75(5):367.

45. Åkerstedt T. Psychosocial stress and impaired sleep. Scand J Work Environ Health 2006;32(6): 493-501.

46. Irwin M, Clark C, Kennedy B, et al. Nocturnal catecholamines and immune function in insomniacs, depressed patients, and control subjects. Brain Behav Immun 2003;17(5):365-72.

47. Vgontzas AN, Chrousos GP. Sleep, the hypothalamic-pituitary-adrenal axis, and cytokines: multiple interactions and disturbances in sleep disorders. Endocrinol Metab Clin North Am 2002; 31(1):15-36.

48. Sephton SE, Lush E, Dedert EA, et al. Diurnal cortisol rhythm as a predictor of lung cancer survival. Brain Behav Immun 2013;30(SUPPL): S163-70.

49. Akıncı T, Melek Başar H. Relationship between sleep quality and the psychological status of patients hospitalised with COVID-19. Sleep Med 2021; 80(January): 167-70.

50. Reiter RJ, Abreu-Gonzalez P, Marik PE, et al. Therapeutic Algorithm for use of melatonin in patients with COVID-19. Front Med (Lausanne) 2020;7:226.

51. Lange T, Dimitrov S, Born J. Effects of sleep and circadian rhythm on the human immune system: annals of the New York Academy of Sciences. Ann N Y Acad Sci 2010;1193:48-59.

52. Besedovsky L, Lange T, Haack M. The sleepimmune crosstalk in health and disease. Physiol Rev 2019;99(3):1325-80.

53. Teixeira KRC, dos Santos CP, de Medeiros LA, et al. Night workers have lower levels of antioxidant defenses and higher levels of oxidative stress damage when compared to day workers. Scientific Rep 2019;9(1):2021.

54. Maas MB, Kim M, Malkani RG, et al. Obstructive sleep apnea and risk of COVID-19 infection, hospitalization and respiratory failure. Sleep Breath 2020;25(2):1155-7.

55. Johnson KG, Sullivan SS, Nti A, et al. The impact of the COVID-19 pandemic on sleep medicine practices. J Clin Sleep Med 2021;17(1):79-87.

56. Iwu CJ, Iwu CD, Wiysonge CS. The occurrence of Iong COVID: a rapid review. Pan Afr Med J 2021; $38: 65$.

57. Chau SWH, Wong OWH, Ramakrishnan R, et al. History for some or lesson for all? A systematic review and meta-analysis on the immediate and long-term mental health impact of the 2002-2003 Severe Acute Respiratory Syndrome (SARS) outbreak. BMC Public Health 2021;21(1):670.

58. Benedict C, Cedernaes J. Could a good night's sleep improve COVID-19 vaccine efficacy? Lancet Respir Med 2021;9(5):447-8.

59. Kar A, Saxena K, Goyal A, et al. Assessment of obstructive sleep apnea in association with severity of COVID-19: a prospective observational study. Sleep Vigil 2021;5(1):111-8.

60. Lockhart SM, O'Rahilly S. When two pandemics meet: why is obesity associated with increased COVID-19 Mortality? Med (N Y) 2020;1(1):33-42.

61. force AC-t. Considerations for the practice of sleep medicine during COVID-19. Darian (Iran): American Academy of Sleep Medicine; 2021. Available at: https://aasm.org/covid-19-resources/considerationspractice-sleep-medicine/. 
62. Gupta R, Kumar VM, Tripathi M, et al. Guidelines of the Indian society for sleep research (ISSR) for practice of sleep medicine during COVID-19. Sleep Vigil 2020;1-12.

63. Schiza S, Simonds A, Randerath W, et al. Sleep laboratories reopening and COVID-19: a European perspective. Eur Respir J 2021;57(3):2002722.

64. Crew EC, Baron KG, Grandner MA, et al. The society of behavioral sleep medicine (SBSM) COVID-19 task force: objectives and summary recommendations for managing sleep during a pandemic. Behav Sleep Med 2020;18(4):570-2.

65. Ayas NT, Fraser KL, Giannouli E, et al. Helping Canadian health care providers to optimize Sleep Disordered Breathing management for their patients during the COVID-19 pandemic. Can J Respir Crit Care Sleep Med 2020;4(2):81-2.

66. Insalaco G, Farra FD, Braghiroli A, et al. Sleep breathing disorders in the COVID-19 Era: Italian Thoracic Society Organizational Models for a correct approach to diagnosis and treatment. Respiration 2020;99(8):690-4.

67. Wilson KC, Kaminsky DA, Michaud G, et al. Restoring pulmonary and sleep services as the
COVID-19 pandemic lessens. From an Association of Pulmonary, Critical Care, and Sleep Division Directors and American Thoracic Societycoordinated Task Force. Ann Am Thorac Soc 2020; 17(11):1343-51.

68. Stern M, Wagner MH, Thompson LA. Current and COVID-19 challenges with childhood and adolescent sleep. JAMA Pediatr 2020;174(11):1124.

69. Kelley GA, Kelley KS. Exercise and sleep: a systematic review of previous meta-analyses. J Evid Based Med 2017;10(1):26-36.

70. Rizzo D, Libman E, Baltzan M, et al. Impact of the COVID-19 pandemic on obstructive sleep apnea: recommendations for symptom management. J Clin Sleep Med 2021;17(3):429-34.

71. Riemann D, Baglioni C, Bassetti C, et al. European guideline for the diagnosis and treatment of insomnia. J Sleep Res 2017;26(6):675-700.

72. Sateia MJ, Buysse DJ, Krystal AD, et al. Clinical practice guideline for the pharmacologic treatment of chronic insomnia in adults: an American Academy of sleep medicine clinical practice guideline. J Clin Sleep Med 2017;13(2):307-49. 